\title{
LETTERS
}

\section{PHAC and a national suicide prevention strategy}

A recent editorial calling for a national suicide prevention strategy, ${ }^{1}$ recommended to "plan the broader infrastructure that is required to address properly what has become a national public health crisis." The Quebec Network on Suicide, Mood Disorders and Related Disorders has proposed several actions based on the Public Health Agency of Canada's (PHAC) engagement with surveillance infrastructures and mental health literacy.

First, other countries audit suicides to useful effect and Canada should follow suit. ${ }^{2}$ An audit of all suicides in New Brunswick identified shortfalls in services in two-thirds of the cases examined, and lead to recommendations for improved population-based literacy efforts, better treatment of mental health and substance misuse and improved coordination of tertiary mental health and addiction services with emergency departments. ${ }^{3}$

Canada should link its mental disorders and its chronic disease surveillance systems to monitor changes in key quality indicators. This is feasible, as shown by the Welsh Public Health Agency, in collaboration with academic departments. ${ }^{4}$ The Canadian Chronic Disease Surveillance System run by $\mathrm{PHAC}$ has similar linked health administrative databases, with each province regularly providing aggregate data.

There should be formal assessment of the efficacy of existing mental health literacy programs in Canada to identify components to retain for a national mental health literacy program for all youth.

More evidence is needed to see if school-based mental health literacy programs are effective in reducing suicidality on a population level.5,6 Existing programs across Canada should be evaluated and harmonized, tailored to their target communities, and adapted to include components that can address the specific needs of Aboriginal communities.

We agree that a national suicide prevention strategy be undertaken by PHAC. This includes building on the successes of previous initiatives to provide the most robust, evidence-based response to this critical public health issue.

\section{Alain Lesage MD}

Co-director

\section{Gustavo Turecki MD PhD}

Director

\section{Sylvanne Daniels MSc PhD}

Coordinator

Quebec Network on Suicide, Mood Disorders

and Related Disorders, Montréal, Que.

- Cite as: CMAJ 2017 January 30;189:E169. doi: $10.1503 / \mathrm{cmaj} .732474$

\section{References}

1. Eggertson L, Patrick K. Canada needs a national suicide prevention strategy. CMAJ 2016;188: E309-10.

2. While D, Bickley $\mathrm{H}$, Roscoe A, et al. Implementation of mental health service recommendations in England and Wales and suicide rates, 19972006: a cross-sectional and before-and-after observational study. Lancet 2012;379:1005-12.

3. Lesage A, Seguin M, Guy A, et al. Systematic services audit of consecutive suicides in New Brunswick: the case for coordinating specialist mental health and addiction services. Can J Psychiatry 2008;53:671-8.

4. John A, Dennis M, Kosnes L, et al. Suicide Information Database-Cymru: a protocol for a population-based, routinely collected data linkage study to explore risks and patterns of healthcare contact prior to suicide to identify opportunities for intervention. BMJ Open 2014;4:e006780.

5. Zalsman G, Hawton K, Wasserman D, et al. Suicide prevention strategies revisited: 10-year systematic review. Lancet Psychiatry 2016;3:646-59.

6. Wasserman D, Hoven CW, Wasserman C, et al. School-based suicide prevention programmes: the SEYLE cluster-randomised, controlled trial. Lancet 2015;385:1536-44. 